\title{
A Gland of Many Uses: A Diversity of Compounds In The Labial Glands of The Bumble Bee Bombus Impatiens Suggests Multiple Signaling Functions
}

\section{Margarita Orlova}

Penn State College of Agricultural Sciences: The Pennsylvania State University College of Agricultural Sciences Gabriel Villar

Penn State College of Agricultural Sciences: The Pennsylvania State University College of Agricultural Sciences Abraham Hefetz

Tel Aviv University Department of Zoology

Jocelyn G. Millar

University of California Riverside

Etya Amsalem ( $D$ eua6@psu.edu )

Penn State College of Agricultural Sciences: The Pennsylvania State University College of Agricultural Sciences https://orcid.org/0000-0001-7935-049X

\section{Research Article}

Keywords: Cephalic labial glands, social insects, reproduction, sex pheromones, signals

Posted Date: July 15th, 2021

DOI: https://doi.org/10.21203/rs.3.rs-689566/v1

License: (a) (1) This work is licensed under a Creative Commons Attribution 4.0 International License. Read Full License

Version of Record: A version of this preprint was published at Journal of Chemical Ecology on March 12th, 2022. See the published version at https://doi.org/10.1007/s10886-022-01348-4. 


\section{Abstract}

Communication in social insect colonies depends on signals accurately reflecting the identity and physiological state of the individuals. Such information is coded by the products of multiple exocrine glands, and the resulting blends reflect the species, sex, caste, age, task, reproductive status, and health of an individual, and may also contain caste-specific pheromones regulating the behavior and physiology of other individuals.

Here we examined the composition of labial gland secretions in females of the bumble bee Bombus impatiens, of different castes, social condition, age, mating status, and ovarian activation. We show that active queens, gynes, and workers each produce caste-specific compounds that may serve different communicative functions. The composition and amounts of wax esters, mostly octyl esters produced by active queens, differed significantly between castes, mating, and social conditions, suggesting a social signaling role. Farnesyl esters were predominant in gynes and peaked at optimal mating age (6-10 days), suggesting their possible roles as sex pheromone components. Reproductive status of females and age across castes was reflected by the ratio between short- and long-chain hydrocarbons, suggesting that these compounds may serve as fertility signals. Our findings overall suggest that the labial gland composition in $B$. impatiens reflects different facets of female bee physiology. While further bioassays are required to determine the function of these compounds, they are likely to have an important role in the communication between individuals and to the cohesion of the social structure.

\section{Introduction}

Insect societies rely on chemical signaling for regulating diverse activities ranging from foraging to reproduction, and the outcome of communication depends heavily on how accurately the signals reflect the identity and physiological state of the individuals. Semiochemicals specific to species, caste, age, task, reproductive status, and social status have been identified in numerous eusocial species and mapped to diverse glandular origins (Amsalem 2020; Billen and Šobotník 2015; Blomquist and Bagnères 2010; Keeling et al. 2004; Stokl and Steiger 2017). Some exocrine glands are specific to certain taxa, whereas others are shared across taxa. These ubiquitous glands in insects are useful tools to study the emergence of new signaling functions across species and levels of social organization.

The labial glands are an outstanding example of such glands. Together with the mandibular, hypopharyngeal, and maxillary glands, they constitute the salivary gland complex of insects. The labial glands have thoracic and cephalic compartments, located, respectively, in the thorax and the head (Poiani and Cruz-Landim 2009). These two compartments have the same origin and also likely share the same secretion in species that have a salivary pouch at the intersection of the compartments (Bombus and Meliponinae genera) (Poiani and Cruz-Landim 2010), making this gland suitable for examining functions associated with social signaling across insect species. Some species (e.g., Apis mellifera) lack this pouch and it is still debatable whether the secretion is the same (Katzav-Gozansky et al. 2001) or different (Poiani and Cruz-Landim 2010) between the two compartments, but the cephalic labial glands are well-developed in eusocial species of the subfamily Apinae in which the secretion is assumed to be associated with social roles (Poiani and Cruz-Landim 2010).

The thoracic part of the labial gland is ubiquitous across insect orders with the notable exception of Coleoptera and has been studied primarily in the context of larval feeding, digestion, and silk production (Afshar et al. 2013; 
Musser et al. 2006; Sehnal and Sutherland 2008), with the main components of the larval glandular secretion being various proteins and digestive enzymes (Rivera-Vega et al. 2018). In social insects, studies have focused mostly on the cephalic labial secretion which have various social roles. In the honey bee, the secretions (mostly hydrocarbons) of both the cephalic and thoracic compartments of the glands were found to be similar. The secretions were suggested to be associated with worker tasks, but their functional role was not examined (Katzav-Gozansky et al. 2001). In stingless bees, the cephalic labial glands contain a variety of wax-type esters and terpenes that serve as trail pheromones (Jarau et al. 2006; Stangler et al. 2009), and geraniol, the main compound in the secretions of nurse workers, was found to increase the proportion of larvae differentiating into queens (Jarau et al. 2010). In bumble bees, the cephalic labial gland secretions have been extensively studied in males of various species and in females of Bombus terrestris. Males produce various terpenes and fatty alcohols that serve for territory marking (Appelgren et al. 1991; Svensson and Bergström 1977; Valterova et al. 2019), and the secretion is highly variable across species and is often used as a chemotaxonomic tool to distinguish between cryptic species (Bertsch et al. 2005). In females, the cephalic labial glands of $B$. terrestris demonstrate quantitative differences in the amounts of fatty acid dodecyl esters between queens and workers. These esters are produced in larger quantities by sterile compared to fertile females in both castes (Amsalem et al. 2014). Finally, in termites, studies have focused on the thoracic labial gland secretions that contain a variety of caste-specific defensive compounds, most of them highly volatile such as pyrazines and benzoic acid (Sillam-Dussès et al. 2012), but also non-volatile food-marking pheromones (Reinhard and Kaib 1995). Although limited to a small number of species, these studies emphasize the social roles of the labial gland products, particularly the cephalic part, in social insects.

Bumble bees are an interesting group for the study of labial gland composition and function. In these species, queens experience both a solitary and a social phase during their life cycle. Newly-emerged queens (gynes) are produced in late summer at the end of the colony annual life cycle. They leave their natal colony and mate before entering a lengthy winter-diapause (Alford 1969). In spring, upon emerging from diapause, they found a nest and live a solitary lifestyle until the first worker emerges. Following that, the queen monopolizes worker reproduction, but only for a short period. Workers retain the ability to reproduce and challenge the queen's reproductive monopoly towards the end of the colony life cycle (Amsalem et al. 2015; Duchateau and Velthuis 1988). Examining the labial gland composition during the transitions in reproductive roles throughout the life cycles of queens and workers may shed light on the function of the gland products, and adaptive changes that the glandular secretion has acquired.

Here we examined the cephalic labial gland secretion across different castes, social conditions, ages, and life stages in the bumble bee Bombus impatiens. Previous studies have shown that despite similarities in the life cycles of $B$. terrestris and B. impatiens, the chemical secretions of Dufour's gland and the cuticular lipid compositions are different (Amsalem et al. 2014; Amsalem et al. 2009; Derstine et al. 2021; Orlova et al. 2020). We examined the composition of the cephalic labial gland contents in gynes and active queens, and in workers under queenright and queenless conditions, across different ages. We discuss possible functions of these secretions in B. impatiens females.

\section{Materials And Methods}

Bumble Bee Rearing. Source colonies for experimental bees were obtained from Koppert Biological Systems (Howell, Michigan, USA) or Biobest Canada Ltd. (Leamington, Ontario, Canada). They were approximately 3-4 
wk old with less than 30 workers each, a queen, and all stages of brood. Colonies were maintained in closed 30 $\times 30 \times 22.5 \mathrm{~cm}$ nest-boxes in a growth chamber at a temperature of $28-30^{\circ} \mathrm{C}, 60 \%$ relative humidity, and constant darkness, and were supplied ad libitum with a $60 \%$ sugar solution and honeybee-collected pollen (Koppert Biological Systems, Howell, Michigan, USA). Queens and workers used in the study were the same as in (Derstine et al. 2021). Briefly, all workers were collected upon emergence ( $<24 \mathrm{hr}$ old) from 7 colonies before the colonies produced gynes and males. Newly emerged workers were individually marked the time of collection and randomly assigned to one of three treatments: queenright ( $Q R, n=70)$, queenless $(Q L, n=70)$, and queenless broodless $(Q L B L, n=70)$. QR workers were returned to their natal $Q R$ colony until they reached their respective age, while QL and QLBL workers were housed in plastic cages (11 cm diameter $\times 7 \mathrm{~cm}$ height) in groups of 3-6 workers without a queen until they reached the desired age of sampling. Queenless groups of workers typically lay eggs within 6-8d (Amsalem et al. 2015), and because the presence of brood affects worker reproduction (Starkey et al. 2019), we included a group without brood. In the QL groups, eggs laid by workers were left intact, while in the QLBL groups, eggs laid by workers were removed daily. We collected 5 workers of each age (days 114 ) in each treatment (70 workers/treatment). All workers were stored at $-80^{\circ} \mathrm{C}$ until dissection. Twenty active queens that were all mated and laying eggs (hereafter, "active queens") were obtained from twenty full-sized colonies with $>100$ workers. These queens were several months old and were actively producing female workers prior to sampling. Newly emerged, unmated queens (hereafter "gynes"; $n=20$ ) were collected from 3 colonies. They were separated from their natal colonies upon emergence to prevent mating, housed in small cages in groups of 3-5 gynes and sampled at 4 time points: $3,6,10$, and $14 \mathrm{~d}$ after emergence (5/time point).

Ovarian Activation. Ovaries were dissected under a stereomicroscope in distilled water, and the largest three terminal oocytes across both ovaries (at least one from each ovary) were measured with an eyepiece micrometer. The mean of these three oocyte measurements was recorded as mean terminal oocyte size. Ovaries were classified into 4 categories based on their developmental stage: 1 - undeveloped ovaries (oocytes $<1 \mathrm{~mm}$ ), 2 - partial development (1-2 mm), 3 - advanced development (2-3 mm) and 4- full development (>3mm).

Preparation and Analysis of Labial Gland Extracts. Both cephalic labial glands were dissected out of the head capsule by opening the sclerotized cuticle of the head capsule with a forceps and separating the two clusters of gland acini (i.e., small saclike cavities that form the glands) from the surrounding tissue using a fine forceps. The clusters of acini were then placed in a vial with $50 \mu \mathrm{l}$ hexane with $100 \mathrm{ng}$ pentadecane as an internal standard. The vials were stored at $-20^{\circ} \mathrm{C}$. Prior to GC analysis, samples were evaporated to a volume of $10 \mu \mathrm{l}$, of which $1 \mu$ l was chemically analyzed.

Extracts were analyzed with an Agilent 7890A GC equipped with a HP-5ms column $(0.25 \mathrm{~mm}$ id $\times 30 \mathrm{~m} \times 0.25$ $\mu \mathrm{m}$ film thickness, Agilent, Santa Clara CA, USA) and interfaced to an Agilent 5975C mass spectrometer. Compounds were tentatively identified based on diagnostic ions in the resulting spectra and retention indices relative to straight-chain alkanes. Where possible, identifications were confirmed by matching retention times and mass spectra with those of authentic standards.

Compounds in labial gland extracts were quantified on a Trace $1310 \mathrm{GC}$ (Thermo Fisher, Waltham, MA, USA) equipped with a flame-ionization detector (FID) and a TG-5MS column $(0.25 \mathrm{~mm}$ id $\times 30 \mathrm{~m} \times 0.25 \mu \mathrm{m}$ film thickness, Thermo Fisher). The temperature program was $60^{\circ} \mathrm{C}$ to $120^{\circ} \mathrm{C}$ at $15^{\circ} \mathrm{C} / \mathrm{min}$, then $4^{\circ} \mathrm{C} / \mathrm{min}$ to $300^{\circ} \mathrm{C}(5$ min hold). The injector port and FID were held at $250^{\circ} \mathrm{C}$ and $320^{\circ} \mathrm{C}$, respectively. 
Synthesis of Ester Standards. Approximately 40 wax esters and terpenoid esters were synthesized by one of three methods, as represented by the following examples. A full list of the esters, and the methods used to synthesize and purify each one is provided in Table 1. Depending on their properties, synthesized compounds were purified by one or more of vacuum flash chromatography, vacuum distillation, or low-temperature recrystallization (see Table 1). 
Table 1

A list of the ester standards and the methods used to synthesize and purify them. Depending on their properties, synthesized compounds were purified by one or more of vacuum flash chromatography, vacuum distillation, or low-temperature recrystallization. Vac flash = vacuum flash chromatography on silica gel, eluting with 7.5\% EtOAc in hexane; Kugel = Kugelrohr distillation under vacuum; Xtal = recrystallization from the specified solvent at the indicated temperature.

\begin{tabular}{|c|c|c|}
\hline Compound & Synthesis method & Purification* \\
\hline Octyl decanoate & $\mathrm{B}$ & Vac flash, Kugel 120 \\
\hline Octyl myristate & $A$ & Vac flash \\
\hline Octyl palmitate & A & Vac flash, Xtal $-20^{\circ} \mathrm{C}$, acetone \\
\hline Octyl stearate & $A$ & Vac flash Xtal $-20^{\circ} \mathrm{C}$, acetone \\
\hline Octyl oleate & $A$ & Vac flash \\
\hline Decyl octanoate & $\mathrm{B}$ & Kugel bp $125^{\circ} \mathrm{C} / 0.35 \mathrm{~mm} \mathrm{Hg}$ \\
\hline Decyl myristate & $\mathrm{B}$ & Vac flash, Xtal $-20^{\circ} \mathrm{C}$, acetone \\
\hline Decyl palmitate & A & Vac flash, Xtal $-20^{\circ} \mathrm{C}$, acetone \\
\hline Decyl oleate & A & Vac flash \\
\hline Dodecyl decanoate & $A$ & Vac flash, Kugel $135^{\circ} \mathrm{C} / 0.05 \mathrm{~mm} \mathrm{Hg}$ \\
\hline Dodecyl dodecanoate & $\mathrm{B}$ & Vac Flash, Xtal hex $-20^{\circ} \mathrm{C}$, hexane \\
\hline Dodecyl myristate & $A$ & Vac flash, Xtal acetone, $-20^{\circ} \mathrm{C}$ \\
\hline Dodecyl palmitate & A & Vac flash, Xtal acetone $-20^{\circ} \mathrm{C}$ \\
\hline Dodecyl palmitoleate & $A$ & Vac flash Xtal acetone $-20^{\circ} \mathrm{C}$ \\
\hline Dodecyl oleate & $A$ & Vac flash, Xtal acetone $-20^{\circ} \mathrm{C}$ \\
\hline Dodecyl linoleate & $A$ & Vac flash \\
\hline Tetradecyl palmitoleate & A & Vac flash, Xtal EtOH, $-20^{\circ} \mathrm{C}$ \\
\hline Hexadecyl palmitoleate & A & Xtal EtOH, $4^{\circ} \mathrm{C}$ \\
\hline Hexadecyl oleate & A & Xtal acetone, $4^{\circ} \mathrm{C}$ \\
\hline Eicosanyl oleate & C & Vac flash, Xtal acetone, $-20^{\circ} \mathrm{C}$ \\
\hline Docosanyl oleate & $\mathrm{C}$ & Vac flash, Xtal aetone, $-20^{\circ} \mathrm{C}$ \\
\hline Tetracosanyl oleate & $\mathrm{C}$ & Vac flash, Xtal hexane, $-20^{\circ} \mathrm{C}$ \\
\hline Hexacosanyl oleate & $\mathrm{C}$ & Vac flash, Xtal EtOAc, $4^{\circ} \mathrm{C}$ \\
\hline Oleyl oleate & A & Vac flash \\
\hline Geranyl oleate & A & Vac flash \\
\hline Geranyl linoleate & A & Vac flash \\
\hline Farnesyl Z9,E11-tetradecanoate & $A$ & Vac flash \\
\hline
\end{tabular}




\begin{tabular}{|lll|}
\hline Compound & Synthesis method & Purification* \\
\hline Farnesyl dodecanoate & B & Vac flash \\
\hline Farnesyl myristate & B & Vac flash \\
\hline Farnesyl palmitoleate & A & Vac flash \\
\hline Farnesyl oleate & A & Vac flash, no crystals from acetone at -20 \\
\hline Farnesyl linoleate & A & Vac flask \\
\hline Farnesyl linolenate & A & Vac flash \\
\hline Citronellyl tridecanoate & A & Vac flash \\
\hline Citronellyl palmitoleate & A & Vac flash \\
\hline Citronellyl stearate & A & Vac flash \\
\hline Citronellyl linoleate & A & Vac flash \\
\hline Dihydrofarnesyl myristate & B & Vac flash \\
\hline Dihydrofarnesyl palmitate & A & Vac flash \\
\hline Dihydrofarnesyl palmitoleate & A & Vac flash \\
\hline Dihydrofarnesyl linoleate & B & Vac flash \\
\hline
\end{tabular}

Method A (example, farnesyl linoleate): Farnesol $(0.222 \mathrm{~g}, 1 \mathrm{mmol})$, linoleic acid $(0.281 \mathrm{~g}, 1 \mathrm{mmol}), 3-(3-$ dimethylaminopropyl)-1-ethyl-carbodiimide hydrochloride $(0.384 \mathrm{~g}, 2 \mathrm{mmol})$, and a few crystals of dimethylaminopyridine catalyst were dissolved in $20 \mathrm{ml} \mathrm{CH}_{2} \mathrm{Cl}_{2}$ and stirred overnight at room temp. The following morning, the solvent was removed by rotary evaporation, and the residue was partitioned between hexane and water. The hexane layer was washed sequentially with $1 \mathrm{M}$ aqueous $\mathrm{HCl}$ and brine, dried over anhydrous $\mathrm{Na}_{2} \mathrm{SO}_{4}$, and concentrated. The residue was purified by vacuum flash chromatography on silica gel, eluting with $7.5 \%$ EtOAc in hexane.

Method B (example decyl myristate): Mytristoyl chloride $(1.24 \mathrm{~g}, 5 \mathrm{mmol}$ ) was added by syringe pump over 30 min to a solution of decanol $(0.95 \mathrm{~g}, 6 \mathrm{mmol})$, pyridine $(0.4 \mathrm{~g}, 5 \mathrm{mmol})$ and a few crystals of dimethylaminopyridine catalyst in $25 \mathrm{ml} \mathrm{CH}_{2} \mathrm{Cl}_{2}$ at room temp, and the mixture was stirred overnight. The solvent was then removed by rotary evaporation and the residue was partitioned between water and hexane. The hexane layer was washed successively with $1 \mathrm{M}$ aqueous $\mathrm{HCl}$ and brine, dried over anhydrous $\mathrm{Na}_{2} \mathrm{SO}_{4}$, and concentrated. The residue was purified by vacuum flash chromatography on silica gel, eluting with $7.5 \%$ EtOAc in hexane. The purified compound was then recrystallized from $15 \mathrm{ml}$ acetone at $-20^{\circ} \mathrm{C}$ overnight, filtering the resulting mixture in a cold room, producing the purified compound as low-melting white plates.

Method C (example eicosanyl oleate): A solution of oleic acid $(0.564 \mathrm{~g}, 2 \mathrm{mmol})$, eicosanyl alcohol $(0.54 \mathrm{~g}, 1.8$ $\mathrm{mmol}$ ), and $50 \mathrm{mg}$ p-toluenesulphonic acid in benzene was refluxed for $3 \mathrm{~h}$, removing the water formed with a Dean-Stark trap. The cooled mixture was diluted with hexane, washed twice with saturated aqueous $\mathrm{NaHCO}_{3}$ and once with brine, then dried over anhydrous $\mathrm{Na}_{2} \mathrm{SO}_{4}$ and concentrated. The residue was purified by vacuum 
flash chromatography on silica gel, eluting with 3\% EtOAc in hexane, and the purified ester was recrystallized from hexane at $-20^{\circ} \mathrm{C}$ overnight.

Statistical Analysis. Statistical analyses were performed using SPSS v.21. Discriminant analysis was used to compare chemical profiles in their entirety between groups. Generalized Linear Models analysis (henceforth GLM) was employed to compare percentages of compounds between groups. Robust estimation was used to handle violations of model assumptions (Ghosh and Basu 2016). In all analyses we used treatment group (QL workers, QLBL workers, QR workers, gynes, and active queens) as the main effect followed by post-hoc contrast estimation using the Least Significant Difference (LSD) method. Generalized Linear Mixed Model analysis was performed to assess the effect of oocyte size and categorical factors on relative amounts of major classes of compounds in the gland. Satterthwaite correction was employed to account for small and unequal sample sizes (Loh 1987; Yau and Kuk 2002). Both oocyte sizes and relative amounts of compounds were Z-transformed and Generalized Linear Mixed Model analysis was performed on standardized values (Z-scores) to obtain standardized beta coefficients. Statistical significance was accepted at $a=0.05$.

\section{Results}

Ovarian Activation. As expected, active queens had fully activated ovaries (mean oocyte length, $3.4 \pm 0.04 \mathrm{~mm}$ ) while gynes had inactive ovaries $(0.82 \pm 0.04 \mathrm{~mm})$. In gynes, oocyte size significantly changed with age (GLMM, $\left.F_{3,16}=68.3, p<0.001\right)$, being smallest on day $3(0.54 \pm 0.02 \mathrm{~mm})$ and peaking on day $10(1.03 \pm 0.02 \mathrm{~mm})$. Oocyte size in workers was significantly affected by social condition and age, with a significant interaction between the two $\left(G L M M, F_{13,164}=253.9, p<0.001\right.$ for age, $F_{2,164}=48.73, p<0.001$ for social condition, $F_{26,164}=$ 27.9, $\mathrm{p}<0.0001$ for interaction). QLBL workers had larger oocytes than both QL and QR workers (mean oocyte length, $1.97 \pm 0.15 \mathrm{~mm}$ for QLBL, $1.35 \pm 0.13 \mathrm{~mm}$ for $\mathrm{QL}$ and $1.11 \pm 0.12 \mathrm{~mm}$ for QR groups, $\mathrm{n}=70$ per group) (post-hoc LSD pairwise contrast, $\mathrm{p}<0.001$ ) and QL workers, on average, had larger oocytes than QR workers (post-hoc LSD pairwise contrast, $\mathrm{p}<0.001$ ). Oocyte size in all worker groups started increasing on day 2 and reached a plateau on day 8 (post-hoc LSD pairwise contrast, $p<0.001$ for all comparisons between days $1-8, p$ $>0.05$ for later time points).

Identification of Gland Constituents. The chemical analyses of the cephalic labial glands showed a total of 79 compounds in queens and workers, of which 53 were conclusively or tentatively identified, and 26 remain unknown at present (Table 2). All compounds were used in subsequent discriminant analyses (Fig. 1) but only known compounds were used in further analyses (Figs. 2-4). The main ions of the unknown compounds are provided as supplementary material (Table S1). The secretion was composed mainly of hydrocarbons ranging from 21 to 33 carbons, fatty acids, wax esters, and terpenoid esters. The majority of the compounds were ubiquitous in all female castes, however 38 compounds (20 fully identified) were present only in active queens ( $n=9$ compounds, mostly wax esters), gynes $(n=10$, mostly terpenoids), queens (both active queens and gynes, $n=9$, mostly terpenoids) and workers ( $n=10$, mostly hydrocarbons and wax esters) (Table 2 ). 
Table 2

Relative percentages of compounds in labial gland secretion presented as means \pm SE. For all compounds styled in bold, identification has been confirmed by external standard. UD stands for undetectable. One sample of active queen and 4 samples of workers were not included in the analysis due to a technical issue with these samples.

\begin{tabular}{|c|c|c|c|c|c|c|c|c|}
\hline \multirow{2}{*}{$\begin{array}{l}\text { Compound } \\
\text { name }\end{array}$} & \multirow[t]{2}{*}{ Class } & \multirow{2}{*}{$\begin{array}{l}\text { Rt } \\
\text { (min) }\end{array}$} & \multicolumn{5}{|c|}{ Mean percentage $(\%) \pm$ SE } & \multirow[t]{2}{*}{ Specificity } \\
\hline & & & $\begin{array}{l}\text { Active } \\
\text { queens } \\
\text { (n= } \\
19)\end{array}$ & $\begin{array}{l}\text { Gynes } \\
(n= \\
20)\end{array}$ & $\begin{array}{l}\text { QL } \\
\text { workers } \\
(n=67)\end{array}$ & $\begin{array}{l}\text { QLBL } \\
\text { workers } \\
(n=70)\end{array}$ & $\begin{array}{l}\text { QR } \\
\text { workers } \\
(n=69)\end{array}$ & \\
\hline E- $\beta$-farnesene & terpenoid & 12.10 & UD & $\begin{array}{l}0.79 \\
\pm 0.07\end{array}$ & UD & UD & UD & Gynes \\
\hline myristic acid & $\begin{array}{l}\text { free fatty } \\
\text { acid }\end{array}$ & 17.27 & $\begin{array}{l}0.130 \\
\pm 0.04\end{array}$ & $\begin{array}{l}1.02 \\
\pm 0.12\end{array}$ & UD & UD & UD & Queens \\
\hline $\begin{array}{l}\text { 2- } \\
\text { Heptadecanone }\end{array}$ & ketone & 21.33 & $\begin{array}{l}0.34 \pm \\
0.05\end{array}$ & $\begin{array}{l}0.08 \\
\pm 0.01\end{array}$ & $\begin{array}{l}0.41 \pm \\
0.03\end{array}$ & $\begin{array}{l}0.40 \pm \\
0.02\end{array}$ & $\begin{array}{l}0.55 \pm \\
0.03\end{array}$ & \\
\hline unknown 1 & unknown & 21.59 & $\begin{array}{l}0.09 \pm \\
0.02\end{array}$ & UD & UD & $\begin{array}{l}0.11 \pm \\
0.02\end{array}$ & $\begin{array}{l}0.07 \pm \\
0.01\end{array}$ & \\
\hline unknown 2 & unknown & 21.70 & UD & UD & $\begin{array}{l}0.18 \pm \\
0.02\end{array}$ & $\begin{array}{l}0.18 \pm \\
0.02\end{array}$ & $\begin{array}{l}0.22 \pm \\
0.03\end{array}$ & Workers \\
\hline$\beta$-springene & terpenoid & 21.73 & $\begin{array}{l}0.07 \pm \\
0.02\end{array}$ & $\begin{array}{l}0.07 \\
\pm 0.02\end{array}$ & UD & UD & UD & Queens \\
\hline $\begin{array}{l}\text { methyl } \\
\text { palmitate }\end{array}$ & $\begin{array}{l}\text { methyl/ethyl } \\
\text { ester }\end{array}$ & 21.85 & $\begin{array}{l}0.64 \pm \\
0.15\end{array}$ & $\begin{array}{l}1.22 \\
\pm 0.16\end{array}$ & $\begin{array}{l}1.61 \pm \\
0.37\end{array}$ & $\begin{array}{l}0.88 \pm \\
0.13\end{array}$ & $\begin{array}{l}2.10 \pm \\
0.28\end{array}$ & \\
\hline palmitic acid & $\begin{array}{l}\text { free fatty } \\
\text { acid }\end{array}$ & 22.98 & $\begin{array}{l}0.39 \pm \\
0.11\end{array}$ & $\begin{array}{l}0.53 \\
\pm 0.07\end{array}$ & $\begin{array}{l}0.10 \pm \\
0.01\end{array}$ & $\begin{array}{l}0.04 \pm \\
0.01\end{array}$ & $\begin{array}{l}0.13 \pm \\
0.01\end{array}$ & \\
\hline heneicosane & hydrocarbon & 25.88 & $\begin{array}{l}0.94 \pm \\
0.09\end{array}$ & $\begin{array}{l}1.12 \\
\pm 0.12\end{array}$ & $\begin{array}{l}1.38 \pm \\
0.16\end{array}$ & $\begin{array}{l}1.13 \pm \\
0.12\end{array}$ & $\begin{array}{l}1.25 \pm \\
0.11\end{array}$ & \\
\hline methyl oleate & $\begin{array}{l}\text { methyl/ethyl } \\
\text { ester }\end{array}$ & 26.05 & $\begin{array}{l}0.90 \pm \\
0.26\end{array}$ & $\begin{array}{l}0.08 \\
\pm 0.02\end{array}$ & $\begin{array}{l}0.08 \pm \\
0.01\end{array}$ & $\begin{array}{l}0.07 \pm \\
0.02\end{array}$ & $\begin{array}{l}0.22 \pm \\
0.03\end{array}$ & \\
\hline $\begin{array}{l}\text { oleic acid + } \\
\text { stearic acid }\end{array}$ & $\begin{array}{l}\text { free fatty } \\
\text { acid }\end{array}$ & 28.02 & $\begin{array}{l}9.76 \pm \\
1.29\end{array}$ & $\begin{array}{l}2.20 \\
\pm 0.28\end{array}$ & $\begin{array}{l}2.05 \pm \\
0.22\end{array}$ & $\begin{array}{l}3.20 \pm \\
0.33\end{array}$ & $\begin{array}{l}2.81 \pm \\
0.34\end{array}$ & \\
\hline (Z)-9-tricosene & hydrocarbon & 29.55 & $\begin{array}{l}15.31 \\
\pm 1.52\end{array}$ & $\begin{array}{l}4.18 \\
\pm 0.60\end{array}$ & $\begin{array}{l}24.11 \pm \\
0.67\end{array}$ & $\begin{array}{l}21.79 \pm \\
0.68\end{array}$ & $\begin{array}{l}22.14 \pm \\
0.68\end{array}$ & \\
\hline tricosane & hydrocarbon & 30.10 & $\begin{array}{l}8.28 \pm \\
0.50\end{array}$ & $\begin{array}{l}2.68 \\
\pm 0.30\end{array}$ & $\begin{array}{l}10.50 \pm \\
0.29\end{array}$ & $\begin{array}{l}10.74 \pm \\
0.35\end{array}$ & $\begin{array}{l}8.31 \pm \\
0.20\end{array}$ & \\
\hline $\begin{array}{l}(Z)-9- \\
\text { tetracosene }\end{array}$ & hydrocarbon & 31.55 & $\begin{array}{l}1.17 \pm \\
0.11\end{array}$ & $\begin{array}{l}0.27 \\
\pm 0.03\end{array}$ & $\begin{array}{l}1.51 \pm \\
0.03\end{array}$ & $\begin{array}{l}1.40 \pm \\
0.04\end{array}$ & $\begin{array}{l}1.33 \pm \\
0.03\end{array}$ & \\
\hline tetracosane & hydrocarbon & 32.06 & $\begin{array}{l}0.22 \pm \\
0.02\end{array}$ & $\begin{array}{l}0.05 \\
\pm 0.00\end{array}$ & $\begin{array}{l}0.42 \pm \\
0.01\end{array}$ & $\begin{array}{l}0.43 \pm \\
0.01\end{array}$ & $\begin{array}{l}0.34 \pm \\
0.01\end{array}$ & \\
\hline $\begin{array}{l}(Z)-9- \\
\text { pentacosene }\end{array}$ & hydrocarbon & 33.56 & $\begin{array}{l}18.11 \\
\pm 1.33\end{array}$ & $\begin{array}{l}9.73 \\
\pm 0.86\end{array}$ & $\begin{array}{l}29.91 \pm \\
0.40\end{array}$ & $\begin{array}{l}28.96 \pm \\
0.48\end{array}$ & $\begin{array}{l}25.72 \pm \\
0.51\end{array}$ & \\
\hline
\end{tabular}




\begin{tabular}{|c|c|c|c|c|c|c|c|c|}
\hline \multirow{2}{*}{$\begin{array}{l}\text { Compound } \\
\text { name }\end{array}$} & \multirow[t]{2}{*}{ Class } & \multirow{2}{*}{$\begin{array}{l}\mathrm{Rt} \\
\text { (min) }\end{array}$} & \multicolumn{5}{|c|}{ Mean percentage $(\%) \pm S E$} & \multirow[t]{2}{*}{ Specificity } \\
\hline & & & $\begin{array}{l}\text { Active } \\
\text { queens } \\
(\mathrm{n}= \\
19)\end{array}$ & $\begin{array}{l}\text { Gynes } \\
(n= \\
20)\end{array}$ & $\begin{array}{l}\text { QL } \\
\text { workers } \\
(n=67)\end{array}$ & $\begin{array}{l}\text { QLBL } \\
\text { workers } \\
(n=70)\end{array}$ & $\begin{array}{l}\text { QR } \\
\text { workers } \\
(n=69)\end{array}$ & \\
\hline pentacosane & hydrocarbon & 34.00 & $\begin{array}{l}3.52 \pm \\
0.29\end{array}$ & $\begin{array}{l}1.15 \\
\pm 0.06\end{array}$ & $\begin{array}{l}7.50 \pm \\
0.20\end{array}$ & $\begin{array}{l}7.36 \pm \\
0.20\end{array}$ & $\begin{array}{l}6.06 \pm \\
0.18\end{array}$ & \\
\hline $\begin{array}{l}\text { octyl } \\
\text { palmitoleate + } \\
\text { decyl } \\
\text { myristoleate }\end{array}$ & wax ester & 34.70 & $\begin{array}{l}0.24 \pm \\
0.03\end{array}$ & UD & UD & UD & UD & Active Q \\
\hline $\begin{array}{l}\text { octyl palmitate } \\
+ \text { decyl } \\
\text { myristate }\end{array}$ & wax ester & 35.33 & $\begin{array}{l}1.37 \pm \\
0.20\end{array}$ & UD & UD & UD & UD & Active Q \\
\hline $\begin{array}{l}\text { (Z)-9- } \\
\text { hexacosene }\end{array}$ & hydrocarbon & 35.36 & UD & $\begin{array}{l}0.24 \\
\pm 0.01\end{array}$ & $\begin{array}{l}0.35 \pm \\
0.01\end{array}$ & $\begin{array}{l}0.38 \pm \\
0.02\end{array}$ & $\begin{array}{l}0.33 \pm \\
0.02\end{array}$ & \\
\hline hexacosane & hydrocarbon & 35.91 & $\begin{array}{l}0.07 \pm \\
0.01\end{array}$ & $\begin{array}{l}0.26 \\
\pm 0.03\end{array}$ & $\begin{array}{l}0.11 \pm \\
0.00\end{array}$ & $\begin{array}{l}0.10 \pm \\
0.00\end{array}$ & $\begin{array}{l}0.08 \pm \\
0.00\end{array}$ & \\
\hline $\begin{array}{l}\text { (Z)-9- } \\
\text { heptacosene }\end{array}$ & hydrocarbon & 37.22 & $\begin{array}{l}2.47 \pm \\
0.43\end{array}$ & $\begin{array}{l}4.55 \\
\pm 0.27\end{array}$ & $\begin{array}{l}5.29 \pm \\
0.28\end{array}$ & $\begin{array}{l}5.04 \pm \\
0.31\end{array}$ & $\begin{array}{l}4.62 \pm \\
0.33\end{array}$ & \\
\hline heptacosane & hydrocarbon & 37.63 & $\begin{array}{l}0.70 \pm \\
0.10\end{array}$ & $\begin{array}{l}0.97 \\
\pm 0.08\end{array}$ & $\begin{array}{l}1.72 \pm \\
0.09\end{array}$ & $\begin{array}{l}1.56 \pm \\
0.09\end{array}$ & $\begin{array}{l}1.44 \pm \\
0.09\end{array}$ & \\
\hline octyl oleate & wax ester & 38.62 & $\begin{array}{l}9.38 \pm \\
1.19\end{array}$ & $\begin{array}{l}0.07 \\
\pm 0.02\end{array}$ & $\begin{array}{l}0.13 \pm \\
0.04\end{array}$ & $\begin{array}{l}0.32 \pm \\
0.10\end{array}$ & $\begin{array}{l}0.16 \pm \\
0.03\end{array}$ & \\
\hline unknown 3 & unknown & 38.77 & UD & $\begin{array}{l}0.01 \\
\pm 0.00\end{array}$ & UD & UD & UD & Gynes \\
\hline $\begin{array}{l}\text { dodecyl } \\
\text { myristate }\end{array}$ & wax ester & 38.79 & UD & UD & $\begin{array}{l}0.01 \pm \\
0.00\end{array}$ & $\begin{array}{l}0.02 \pm \\
0.00\end{array}$ & $\begin{array}{l}0.02 \pm \\
0.00\end{array}$ & Workers \\
\hline $\begin{array}{l}\text { decyl palmitate } \\
+ \text { octyl stearate }\end{array}$ & wax ester & 38.88 & $\begin{array}{l}0.32 \pm \\
0.07\end{array}$ & UD & UD & UD & UD & Active Q \\
\hline $\begin{array}{l}\text { (Z)-9- } \\
\text { octacosene }\end{array}$ & hydrocarbon & 38.91 & UD & $\begin{array}{l}0.11 \\
\pm 0.01\end{array}$ & $\begin{array}{l}0.31 \pm \\
0.04\end{array}$ & $\begin{array}{l}0.26 \pm \\
0.03\end{array}$ & $\begin{array}{l}0.26 \pm \\
0.03\end{array}$ & \\
\hline unknown 4 & unknown & 39.06 & UD & $\begin{array}{l}0.17 \\
\pm 0.03\end{array}$ & UD & UD & UD & Gynes \\
\hline farnesyl laurate & terpenoid & 39.41 & $\begin{array}{l}0.14 \pm \\
0.04\end{array}$ & $\begin{array}{l}0.70 \\
\pm 0.10\end{array}$ & UD & UD & UD & Queens \\
\hline octacosane & hydrocarbon & 39.42 & UD & UD & $\begin{array}{l}0.06 \pm \\
0.01\end{array}$ & $\begin{array}{l}0.08 \pm \\
0.01\end{array}$ & $\begin{array}{l}0.04 \pm \\
0.00\end{array}$ & Workers \\
\hline squalene & terpenoid & 39.66 & $\begin{array}{l}0.09 \pm \\
0.01\end{array}$ & UD & $\begin{array}{l}0.11 \pm \\
0.02\end{array}$ & $\begin{array}{l}0.16 \pm \\
0.03\end{array}$ & $\begin{array}{l}0.14 \pm \\
0.02\end{array}$ & \\
\hline $\begin{array}{l}\text { (Z)-9- } \\
\text { nonacosene }\end{array}$ & hydrocarbon & 40.65 & $\begin{array}{l}1.56 \pm \\
0.25\end{array}$ & $\begin{array}{l}2.81 \\
\pm 0.22\end{array}$ & $\begin{array}{l}2.71 \pm \\
0.13\end{array}$ & $\begin{array}{l}2.68 \pm \\
0.18\end{array}$ & $\begin{array}{l}2.38 \pm \\
0.17\end{array}$ & \\
\hline
\end{tabular}




\begin{tabular}{|c|c|c|c|c|c|c|c|c|}
\hline \multirow{2}{*}{$\begin{array}{l}\text { Compound } \\
\text { name }\end{array}$} & \multirow[t]{2}{*}{ Class } & \multirow{2}{*}{$\begin{array}{l}\mathrm{Rt} \\
\text { (min) }\end{array}$} & \multicolumn{5}{|c|}{ Mean percentage $(\%) \pm S E$} & \multirow[t]{2}{*}{ Specificity } \\
\hline & & & $\begin{array}{l}\text { Active } \\
\text { queens } \\
(\mathrm{n}= \\
19)\end{array}$ & $\begin{array}{l}\text { Gynes } \\
(n= \\
20)\end{array}$ & $\begin{array}{l}\text { QL } \\
\text { workers } \\
(n=67)\end{array}$ & $\begin{array}{l}\text { QLBL } \\
\text { workers } \\
(n=70)\end{array}$ & $\begin{array}{l}\text { QR } \\
\text { workers } \\
(n=69)\end{array}$ & \\
\hline nonacosane & hydrocarbon & 40.99 & $\begin{array}{l}0.31 \pm \\
0.04\end{array}$ & $\begin{array}{l}0.24 \\
\pm 0.02\end{array}$ & $\begin{array}{l}0.83 \pm \\
0.03\end{array}$ & $\begin{array}{l}0.77 \pm \\
0.04\end{array}$ & $\begin{array}{l}0.74 \pm \\
0.04\end{array}$ & \\
\hline $\begin{array}{l}\text { geranyl } \\
\text { linoleate }\end{array}$ & terpenoid & 41.73 & UD & $\begin{array}{l}0.45 \\
\pm 0.04\end{array}$ & $\begin{array}{l}0.03 \pm \\
0.00\end{array}$ & $\begin{array}{l}0.03 \pm \\
0.00\end{array}$ & $\begin{array}{l}0.08 \pm \\
0.00\end{array}$ & \\
\hline $\begin{array}{l}\text { farnesyl } \\
\text { myristate }\end{array}$ & terpenoid & 41.75 & $\begin{array}{l}0.20 \pm \\
0.06\end{array}$ & UD & UD & UD & UD & Active Q \\
\hline decyl oleate & wax ester & 41.91 & $\begin{array}{l}3.92 \pm \\
0.52\end{array}$ & UD & $\begin{array}{l}0.06 \pm \\
0.02\end{array}$ & $\begin{array}{l}0.17 \pm \\
0.05\end{array}$ & $\begin{array}{l}0.10 \pm \\
0.02\end{array}$ & \\
\hline $\begin{array}{l}\text { dodecyl } \\
\text { palmitate }\end{array}$ & wax ester & 42.10 & $\begin{array}{l}0.18 \pm \\
0.15\end{array}$ & UD & $\begin{array}{l}0.02 \pm \\
0.00\end{array}$ & $\begin{array}{l}0.03 \pm \\
0.00\end{array}$ & $\begin{array}{l}0.06 \pm \\
0.01\end{array}$ & \\
\hline $\begin{array}{l}\text { dihydrofarnesyl } \\
\text { myristate }\end{array}$ & terpenoid & 42.10 & UD & $\begin{array}{l}0.05 \\
\pm 0.01\end{array}$ & UD & UD & UD & Gynes \\
\hline $\begin{array}{l}\text { (Z)-9- } \\
\text { triacontene }\end{array}$ & hydrocarbon & 42.28 & $\begin{array}{l}0.08 \pm \\
0.02\end{array}$ & $\begin{array}{l}0.13 \\
\pm 0.01\end{array}$ & $\begin{array}{l}0.10 \pm \\
0.01\end{array}$ & $\begin{array}{l}0.10 \pm \\
0.01\end{array}$ & $\begin{array}{l}0.08 \pm \\
0.00\end{array}$ & \\
\hline unknown 5 & unknown & 42.45 & $\begin{array}{l}0.12 \pm \\
0.09\end{array}$ & $\begin{array}{l}0.15 \\
\pm 0.02\end{array}$ & UD & UD & UD & Queens \\
\hline unknown 6 & unknown & 42.56 & $\begin{array}{l}0.06 \pm \\
0.02\end{array}$ & $\begin{array}{l}0.11 \\
\pm 0.03\end{array}$ & UD & UD & UD & Queens \\
\hline triacontane & hydrocarbon & 42.65 & UD & UD & $\begin{array}{l}0.04 \pm \\
0.01\end{array}$ & $\begin{array}{l}0.04 \pm \\
0.01\end{array}$ & $\begin{array}{l}0.06 \pm \\
0.01\end{array}$ & Workers \\
\hline unknown 7 & unknown & 42.7 & $\begin{array}{l}0.05 \pm \\
0.01\end{array}$ & $\begin{array}{l}1.24 \\
\pm 0.18\end{array}$ & UD & UD & UD & Queens \\
\hline $\begin{array}{l}\text { (Z)-9- } \\
\text { hentriacontene }\end{array}$ & hydrocarbon & 44.0 & $\begin{array}{l}1.06 \pm \\
0.17\end{array}$ & $\begin{array}{l}0.80 \\
\pm 0.08\end{array}$ & $\begin{array}{l}1.58 \pm \\
0.10\end{array}$ & $\begin{array}{l}1.65 \pm \\
0.10\end{array}$ & $\begin{array}{l}1.26 \pm \\
0.05\end{array}$ & \\
\hline hentriacontane & hydrocarbon & 44.16 & $\begin{array}{l}0.14 \pm \\
0.02\end{array}$ & $\begin{array}{l}0.03 \\
\pm 0.00\end{array}$ & $\begin{array}{l}0.32 \pm \\
0.01\end{array}$ & $\begin{array}{l}0.29 \pm \\
0.01\end{array}$ & $\begin{array}{l}0.31 \pm \\
0.01\end{array}$ & \\
\hline unknown 8 & unknown & 44.46 & $\begin{array}{l}0.10 \pm \\
0.03\end{array}$ & UD & UD & UD & UD & Active Q \\
\hline unknown 9 & unknown & 44.58 & UD & UD & $\begin{array}{l}0.01 \pm \\
0.00\end{array}$ & $\begin{array}{l}0.03 \pm \\
0.00\end{array}$ & $\begin{array}{l}0.05 \pm \\
0.00\end{array}$ & Workers \\
\hline unknown 10 & unknown & 44.79 & UD & $\begin{array}{l}2.77 \\
\pm 0.33\end{array}$ & UD & UD & UD & Gynes \\
\hline $\begin{array}{l}\text { dihydrofarnesyl } \\
\text { palmitoleate }\end{array}$ & terpenoid & 44.89 & UD & $\begin{array}{l}0.05 \\
\pm 0.01\end{array}$ & UD & UD & UD & Gynes \\
\hline dodecyl oleate & wax ester & 44.89 & $\begin{array}{l}0.52 \pm \\
0.08\end{array}$ & UD & $\begin{array}{l}0.19 \pm \\
0.02\end{array}$ & $\begin{array}{l}0.33 \pm \\
0.04\end{array}$ & $\begin{array}{l}0.43 \pm \\
0.07\end{array}$ & \\
\hline
\end{tabular}




\begin{tabular}{|c|c|c|c|c|c|c|c|c|}
\hline \multirow{2}{*}{$\begin{array}{l}\text { Compound } \\
\text { name }\end{array}$} & \multirow[t]{2}{*}{ Class } & \multirow{2}{*}{$\begin{array}{l}\mathrm{Rt} \\
\text { (min) }\end{array}$} & \multicolumn{5}{|c|}{ Mean percentage $(\%) \pm S E$} & \multirow[t]{2}{*}{ Specificity } \\
\hline & & & $\begin{array}{l}\text { Active } \\
\text { queens } \\
(\mathrm{n}= \\
19)\end{array}$ & $\begin{array}{l}\text { Gynes } \\
(n=) \\
20)\end{array}$ & $\begin{array}{l}\text { QL } \\
\text { workers } \\
(n=67)\end{array}$ & $\begin{array}{l}\text { QLBL } \\
\text { workers } \\
(n=70)\end{array}$ & $\begin{array}{l}\text { QR } \\
\text { workers } \\
(n=69)\end{array}$ & \\
\hline $\begin{array}{l}\text { dihydrofarnesyl } \\
\text { palmitate }\end{array}$ & terpenoid & 45.25 & UD & $\begin{array}{l}0.15 \\
\pm 0.02\end{array}$ & UD & UD & UD & Gynes \\
\hline $\begin{array}{l}\text { dodecyl } \\
\text { linoleate }\end{array}$ & wax ester & 45.35 & UD & UD & $\begin{array}{l}0.03 \pm \\
0.00\end{array}$ & $\begin{array}{l}0.04 \pm \\
0.00\end{array}$ & $\begin{array}{l}0.03 \pm \\
0.00\end{array}$ & Workers \\
\hline $\begin{array}{l}\text { farnesyl } \\
\text { palmitoleate }\end{array}$ & terpenoid & 45.5 & $\begin{array}{l}0.25 \pm \\
0.04\end{array}$ & $\begin{array}{l}3.02 \\
\pm 0.26\end{array}$ & UD & UD & UD & Queens \\
\hline dotriacontane & hydrocarbon & 45.76 & UD & UD & $\begin{array}{l}0.08 \pm \\
0.01\end{array}$ & $\begin{array}{l}0.12 \pm \\
0.02\end{array}$ & $\begin{array}{l}0.19 \pm \\
0.01\end{array}$ & Workers \\
\hline $\begin{array}{l}\text { farnesyl } \\
\text { palmitate }\end{array}$ & terpenoid & 45.88 & $\begin{array}{l}0.18 \pm \\
0.05\end{array}$ & $\begin{array}{l}3.22 \\
\pm 0.35\end{array}$ & UD & UD & UD & Queens \\
\hline unknown 11 & unknown & 46.3 & $\begin{array}{l}0.15 \pm \\
0.09\end{array}$ & $\begin{array}{l}0.11 \\
\pm 0.01\end{array}$ & $\begin{array}{l}0.01 \pm \\
0.00\end{array}$ & $\begin{array}{l}0.10 \pm \\
0.02\end{array}$ & $\begin{array}{l}0.07 \pm \\
0.02\end{array}$ & \\
\hline unknown 12 & unknown & 46.45 & $\begin{array}{l}0.17 \pm \\
0.11\end{array}$ & UD & $\begin{array}{l}0.03 \pm \\
0.00\end{array}$ & $\begin{array}{l}0.07 \pm \\
0.01\end{array}$ & $\begin{array}{l}0.08 \pm \\
0.01\end{array}$ & \\
\hline unknown 13 & unknown & 46.63 & UD & $\begin{array}{l}0.28 \\
\pm 0.03\end{array}$ & UD & UD & UD & Gynes \\
\hline $\begin{array}{l}\text { (Z)-9- } \\
\text { tritriacontene }\end{array}$ & hydrocarbon & 46.86 & $\begin{array}{l}0.11 \pm \\
0.02\end{array}$ & $\begin{array}{l}0.40 \\
\pm 0.05\end{array}$ & $\begin{array}{l}0.19 \pm \\
0.02\end{array}$ & $\begin{array}{l}0.20 \pm \\
0.02\end{array}$ & $\begin{array}{l}0.16 \pm \\
0.01\end{array}$ & \\
\hline $\begin{array}{l}\text { myristoleyl } \\
\text { oleate + oleyl } \\
\text { myristoleate }\end{array}$ & wax ester & 47.68 & $\begin{array}{l}0.58 \pm \\
0.04\end{array}$ & UD & $\begin{array}{l}0.06 \pm \\
0.01\end{array}$ & $\begin{array}{l}0.10 \pm \\
0.02\end{array}$ & $\begin{array}{l}0.15 \pm \\
0.01\end{array}$ & \\
\hline $\begin{array}{l}\text { dihydrofarnesyl } \\
\text { linoleate }\end{array}$ & terpenoid & 47.92 & UD & $\begin{array}{l}1.41 \\
\pm 0.20\end{array}$ & UD & UD & UD & Gynes \\
\hline farnesyl oleate & terpenoid & 48.65 & $\begin{array}{l}1.95 \pm \\
0.33\end{array}$ & $\begin{array}{l}31.43 \\
\pm 2.16\end{array}$ & $\begin{array}{l}0.20 \pm \\
0.02\end{array}$ & $\begin{array}{l}0.29 \pm \\
0.03\end{array}$ & $\begin{array}{l}0.46 \pm \\
0.03\end{array}$ & \\
\hline unknown 14 & unknown & 48.75 & UD & UD & $\begin{array}{l}0.25 \pm \\
0.04\end{array}$ & $\begin{array}{l}0.45 \pm \\
0.06\end{array}$ & $\begin{array}{l}0.84 \pm \\
0.07\end{array}$ & Workers \\
\hline $\begin{array}{l}\text { farnesyl } \\
\text { linoleate }\end{array}$ & terpenoid & 49.5 & $\begin{array}{l}0.38 \pm \\
0.05\end{array}$ & $\begin{array}{l}0.74 \\
\pm 0.04\end{array}$ & UD & UD & UD & Queens \\
\hline unknown 15 & unknown & 49.5 & UD & UD & $\begin{array}{l}0.18 \pm \\
0.05\end{array}$ & $\begin{array}{l}0.19 \pm \\
0.03\end{array}$ & $\begin{array}{l}0.43 \pm \\
0.03\end{array}$ & Workers \\
\hline $\begin{array}{l}\text { hexadecyl } \\
\text { oleate }\end{array}$ & wax ester & 51.86 & $\begin{array}{l}5.92 \pm \\
1.72\end{array}$ & $\begin{array}{l}8.53 \\
\pm 0.95\end{array}$ & $\begin{array}{l}2.60 \pm \\
0.33\end{array}$ & $\begin{array}{l}4.16 \pm \\
0.49\end{array}$ & $\begin{array}{l}7.23 \pm \\
0.45\end{array}$ & \\
\hline unknown 16 & unknown & 52.75 & $\begin{array}{l}2.78 \pm \\
0.75\end{array}$ & $\begin{array}{l}4.13 \\
\pm 0.44\end{array}$ & $\begin{array}{l}1.24 \pm \\
0.16\end{array}$ & $\begin{array}{l}1.82 \pm \\
0.19\end{array}$ & $\begin{array}{l}3.44 \pm \\
0.21\end{array}$ & \\
\hline unknown 17 & unknown & 52.98 & $\begin{array}{l}0.62 \pm \\
0.20\end{array}$ & $\begin{array}{l}0.67 \\
\pm 0.07\end{array}$ & $\begin{array}{l}0.24 \pm \\
0.03\end{array}$ & $\begin{array}{l}0.19 \pm \\
0.02\end{array}$ & $\begin{array}{l}0.31 \pm \\
0.03\end{array}$ & \\
\hline
\end{tabular}




\begin{tabular}{|c|c|c|c|c|c|c|c|c|}
\hline \multirow{2}{*}{$\begin{array}{l}\text { Compound } \\
\text { name }\end{array}$} & \multirow[t]{2}{*}{ Class } & \multirow{2}{*}{$\begin{array}{l}\mathrm{Rt} \\
\text { (min) }\end{array}$} & \multicolumn{5}{|c|}{ Mean percentage $(\%) \pm S E$} & \multirow[t]{2}{*}{ Specificity } \\
\hline & & & $\begin{array}{l}\text { Active } \\
\text { queens } \\
(\mathrm{n}= \\
19)\end{array}$ & $\begin{array}{l}\text { Gynes } \\
(n= \\
20)\end{array}$ & $\begin{array}{l}\text { QL } \\
\text { workers } \\
(n=67)\end{array}$ & $\begin{array}{l}\text { QLBL } \\
\text { workers } \\
(n=70)\end{array}$ & $\begin{array}{l}\text { QR } \\
\text { workers } \\
(n=69)\end{array}$ & \\
\hline unknown 18 & unknown & 53.37 & $\begin{array}{l}0.19 \pm \\
0.04\end{array}$ & UD & UD & UD & UD & Active Q \\
\hline unknown 19 & unknown & 53.62 & $\begin{array}{l}0.11 \pm \\
0.03\end{array}$ & UD & $\begin{array}{l}0.25 \pm \\
0.03\end{array}$ & $\begin{array}{l}0.30 \pm \\
0.08\end{array}$ & $\begin{array}{l}0.48 \pm \\
0.04\end{array}$ & \\
\hline unknown 20 & unknown & 53.85 & UD & $\begin{array}{l}0.17 \\
\pm 0.02\end{array}$ & UD & UD & UD & Gynes \\
\hline unknown 21 & unknown & 54.11 & $\begin{array}{l}1.08 \pm \\
0.34\end{array}$ & $\begin{array}{l}1.61 \\
\pm 0.21\end{array}$ & $\begin{array}{l}0.52 \pm \\
0.06\end{array}$ & $\begin{array}{l}0.66 \pm \\
0.07\end{array}$ & $\begin{array}{l}1.35 \pm \\
0.09\end{array}$ & \\
\hline unknown 22 & unknown & 54.29 & $\begin{array}{l}0.24 \pm \\
0.06\end{array}$ & UD & UD & UD & UD & Active Q \\
\hline unknown 23 & unknown & 54.53 & $\begin{array}{l}0.33 \pm \\
0.03\end{array}$ & UD & UD & UD & UD & Active Q \\
\hline unknown 24 & unknown & 54.67 & $\begin{array}{l}0.21 \pm \\
0.09\end{array}$ & UD & UD & UD & UD & Active Q \\
\hline unknown 25 & unknown & 54.83 & UD & UD & $\begin{array}{l}0.24 \pm \\
0.02\end{array}$ & $\begin{array}{l}0.42 \pm \\
0.04\end{array}$ & $\begin{array}{l}0.44 \pm \\
0.02\end{array}$ & Workers \\
\hline unknown 26 & unknown & 55.05 & UD & $\begin{array}{l}0.14 \\
\pm 0.03\end{array}$ & $\begin{array}{l}0.05 \pm \\
0.01\end{array}$ & $\begin{array}{l}0.01 \pm \\
0.00\end{array}$ & $\begin{array}{l}0.14 \pm \\
0.01\end{array}$ & \\
\hline $\begin{array}{l}\text { oleyl oleate and } \\
\text { other long } \\
\text { esters }\end{array}$ & wax ester & 57.26 & $\begin{array}{l}1.04 \pm \\
0.30\end{array}$ & $\begin{array}{l}2.90 \\
\pm 0.43\end{array}$ & $\begin{array}{l}0.10 \pm \\
0.02\end{array}$ & $\begin{array}{l}0.12 \pm \\
0.01\end{array}$ & $\begin{array}{l}0.30 \pm \\
0.02\end{array}$ & \\
\hline
\end{tabular}

Discriminant Analyses. The cephalic labial gland profiles of all bees were analyzed using discriminant analysis using relative quantities of substances. Four discriminant functions significantly discriminated between gynes, active queens, and workers using this analysis, with the first two functions explaining $99.8 \%$ of the variance. Function 1 (eigenvalue $=2127.76$, canonical correlation $=1$, percent of explained variance $=94.3 \%$; Wilk's $\lambda_{304}<$ $\left.0.0001, \chi^{2}=2841.65, p<0.001\right)$ discriminated between gynes and all other bees and had the highest correlation values with farnesyl and dihydrofarnesyl esters, while function 2 (eigenvalue $=123.29$, canonical correlation $=$ 0.99 , percent of explained variance $=5.5 \%$; Wilk's $\lambda_{225}=0.001, \chi^{2}=1381.79, p<0.001$ ), discriminated between active queens and all other bees, and had the highest correlation values with wax esters, particularly octyl esters (Fig. 1A).

Because large differences between castes may have obscured differences between treatments and ages in workers, we analyzed these data separately. Two discriminant functions significantly discriminated between QR, $\mathrm{QL}$, and QLBL treatments in this analysis. Function 1 (eigenvalue $=4.626$, canonical correlation $=0.91$, percent of explained variance $=69.3 \%$; Wilk's $\lambda_{100}=0.059, \chi^{2}=469.74, p<0.001$ ) discriminated between QR and QLBL workers and had the highest correlation values with methyl palmitate, dodecyl linoleate, geranyl linoleate, octacosane, (Z)-9-triacontene and an unknown compound characterized by a base peak at $m / z=95$ (unknown 
26 in Table 2) while function 2 (eigenvalue $=2.04$, canonical correlation $=0.82$, percent of explained variance $=$ $30.6 \%$; Wilk's $\left.\lambda_{49}=0.32, \chi^{2}=183.8, p<0.001\right)$, discriminated between QL workers and the other two groups, and had the highest correlation values with farnesyl linoleate, hexadecyl oleate, oleyl oleate, and other long-chain wax esters as well as two other unknown compounds characterized by a base peak at $m / z=95$ (unknowns 14 and 16 in Table 2) (Fig. 1B).

Thirteen discriminant functions significantly discriminated between workers of different age groups, with the first two functions explaining $83.2 \%$ of the variance. Function 1 (eigenvalue $=23.65$, canonical correlation $=0.98$, percent of explained variance $=67.3 \%$; Wilk's $\left.\lambda_{650}<0.0001, \chi^{2}=1519.26, p<0.001\right)$ discriminated between all ages from 1 to 14 days in workers, while function 2 (eigenvalue $=5.58$, canonical correlation $=0.92$, percent of explained variance $=15.9 \%$; Wilk's $\lambda_{588}=0.002, \chi^{2}=1006.8, p<0.001$ ), discriminated between workers at 3-6 days of age and workers at younger and older ages. Both functions had the highest correlation values with the most abundant hydrocarbon compounds, with short- and long-chain hydrocarbons displaying negative and positive correlation coefficients, respectively (Fig. 1C).

The two discriminant functions significantly discriminated between workers with oocytes at different stages of activation with the first two functions explaining $95 \%$ of the variance in reproduction. Function 1 (eigenvalue $=$ 3.72 , canonical correlation $=0.89$, percent of explained variance $=62.7 \%$; Wilk's $\lambda_{150}=0.056, \chi^{2}=475.45, p<$ 0.001 ) separated workers with undeveloped ovaries (stage 1) from all other workers and had highest correlation values with the major hydrocarbon compounds (heneicosane, tricosane, tetracosane, heptacosane, nonacosane and the corresponding alkenes) with short- and long-chain hydrocarbons displaying negative and positive correlation coefficients, respectively.

Based on the discriminant analysis information, further analyses of the labial gland secretions were done using three major classes of compounds: 1) terpenoid compounds, comprising farnesene, farnesyl esters, dihydrofarnesyl esters, and geranyl esters, 2) wax esters, with the alcohol moiety chain lengths ranging from 8 to 18 carbons and the acid moiety chain lengths ranging from 14 to 22 carbons, 3) hydrocarbons with chain lengths ranging from 21 to 33 carbons. The relative proportion of each compound class in the total secretion was calculated and used in further analyses. The ratio of short- ( $\leq 24$ carbons) to long-chain hydrocarbons ( $\geq$ 26 carbons) was also calculated.

Terpenoid Components. GLM analysis revealed that the proportions of terpenoid components differed significantly between all groups and were highest in gynes, where they comprised up to $68 \%$ of the total secretion, and lowest in QL workers ( $0.1 \%$ of the total secretion) (GLM, Wald $\chi^{2}{ }_{4}=327.67, p<0.0001$ for group, $p$ $<0.0001$ for all post hoc comparisons) (Fig. 2A). Among gynes of different ages, terpenoid compound proportions peaked on days 6 and 10 and declined on day 14 , without covariance with oocyte size or interaction between oocyte size and age (GLMM, $F_{3,12}=9.19, p=0.002$ for age, $F_{1,12}=1.49, p=0.24$ for oocyte size, $F_{3,12}=$ $0.4, p=0.75$ for interaction) (Fig. 2B).

Wax Ester Components. The proportion of wax esters was highest in active queens (on average 23\%) and lowest in QL workers (on average 2.9\%) (GLM, Wald $\chi^{2}{ }_{4}=211.44, p<0.0001$ for group, $p<0.05$ for all post hoc comparisons). However, the composition of wax esters differed between groups, with octyl esters being almost exclusively present in active queens and dodecyl ester proportions being highest in active queens, QR, and QLBL

Page $14 / 23$ 
workers and undetectable in gynes, which almost exclusively produced long-chain esters (> 32 carbons in total) (Fig. 3). Wax ester proportion in gynes was not significantly explained by either age or by oocyte size (GLMM, $F_{3,12}=1.82, p=0.19$ for age, $F_{1,12}=1.1, p=0.31$ for oocyte size, $F_{3,12}=0.47, p=0.70$ for interaction).

Following our finding on caste differences in abundance of different wax esters we performed a discriminant analysis based solely on ester compounds. Four discriminant functions significantly discriminated between gynes, active queens, and workers using this analysis, with the first two functions explaining $97 \%$ of the variance. Function 1 (eigenvalue $=12.55$, canonical correlation $=0.96$, percent of explained variance $=83.5 \%$; Wilk's $\left.\lambda_{56}=0.016, \chi^{2}=910.05, p<0.001\right)$ discriminated between active queens and all other bees and had highest correlation values with octyl esters, while function 2 (eigenvalue $=2.02$, canonical correlation $=0.81$, percent of explained variance $=13.5 \%$; Wilk's $\left.\lambda_{39}=0.22, \chi^{2}=310.01, p<0.001\right)$, discriminated between gynes and all other bees and had highest correlation values with very long chain esters ( $>32$ carbons). Workers of different treatment groups could not be discriminated based on ester composition.

Differences in Compound Classes Between Workers of Different Ages and Treatments. Based on the results of the discriminant analysis, we tested whether treatment group, age, and ovary size predicted the relative proportion of wax esters and the series of unknown compounds with $m / z 95$ mass spectral base peak. Wax ester proportion was significantly predicted by age and treatment group, being highest in QR workers and at later ages (day 11 and later) but not by ovary size, with significant interaction between age and treatment and age and ovary size (GLMM, $F_{13,148}=3.72, p<0.0001$ for age, $F_{2,148}=31.11, p<0.0001$ for treatment, $F_{26,148}=3.75, p$ $<0.0001$ for interaction between age and treatment, $F_{1,148}=1.13, p=0.28$ for covariance with ovary size, $F_{2,148}$ $=1.76, p=0.17$ for interaction between treatment and ovary size and $F_{13,148}=1.8, p=0.047$ for interaction between age and ovary size). The proportion of unidentified compounds with $m / z=95$-base peak compounds was significantly predicted by age, treatment group (highest in QR workers) and ovary size, with significant interaction between age and treatment $\left(G L M M, F_{13,148}=3.79, p<0.0001\right.$ for age, $F_{2,148}=38.99, p<0.0001$ for treatment, $F_{26,148}=3.97, p<0.0001$ for interaction between age and treatment, $F_{1,148}=10.88, p=0.001$ for covariance with ovary size, $F_{2,148}=1.45, p=0.23$ for interaction between treatment and ovary size and $F_{13,148}$ $=1.73, p=0.05$ for interaction between age and ovary size).

Hydrocarbon Composition and Ovarian Development. In line with a previous study (Orlova et al. 2020), the shortto long-chain hydrocarbon ratio was highest in active queens $(5.2 \pm 0.16)$ and lowest in gynes $(0.82 \pm 0.09)$ (GLM, Wald $\chi_{4}^{2}=295.66, p<0.0001$ for group, post-hoc LSD: $p<0.0001$ for active queen vs. other groups, $p<$ 0.0001 for gyne vs. other groups, $p>0.05$ for comparisons between worker treatments). In workers, short- to long-chain hydrocarbon ratio was on average $3.2 \pm 0.08$ and was significantly predicted by age and treatment group and ovary size, peaking on day 8 and being initially higher in QLBL and QL workers, and then in QR workers at later ages with significant interaction between age and treatment and age and ovary size (GLMM, $F_{13,148}=7.90, p<0.0001$ for age, $F_{2,148}=11.61, p<0.0001$ for treatment, $F_{26,148}=5.61, p<0.0001$ for interaction between age and treatment, $F_{1,148}=50.91, p<0.0001$ for covariance with ovary size, $F_{2,148}=2.08, p=0.12$ for interaction between treatment and ovary size and $F_{13,148}=6.86, p<0.0001$ for interaction between age and ovary size). In gynes, short- to long-chain hydrocarbon ratio peaked on day 14 and displayed no covariance with oocyte size, but there was significant interaction between oocyte size and age (GLMM, $F_{3,12}=51.04, p<0.0001$ for age, $F_{1,12}=3.2, p=0.098$ for oocyte size, $F_{3,12}=12.16, p=0.001$ for interaction). When the relationship 
between short to long $\mathrm{CHC}$ ratio was analyzed separately using regression curve estimation, polynomial regression with cubic fit proved the best fitting curve $\left(R=0.57, R^{2}=0.325, F_{3,202}=32.39, p<0.0001\right)$ (Fig. 4).

\section{Discussion}

Our analysis of the cephalic labial gland secretions revealed a great diversity of compounds representing a number of different chemical classes. This structural diversity, and the substantial differences in composition between bees of differing caste, age, and social condition allude to diverse roles played by the different compounds. Some of these differences, such as the abundance of terpenoids in gynes and the octyl esters in queens, parallel those found in other secretions of B. impatiens and B. terrestris (Amsalem et al. 2014; Amsalem et al. 2009; Derstine et al. 2021). Overall, we showed strong associations of terpenoid compounds with caste and mating status, of esters with social condition, and of the hydrocarbon profile with reproductive status.

Terpenoid compounds were predominant in gynes. These compounds comprised $40-60 \%$ of the total secretion, and their amounts peaked in gynes aged 6 to 10 days, coinciding with the age range optimal for mating (Treanore, Barie, Derstine, Gadebusch, Orlova, Porter, Purnell and Amsalem, submitted). This finding suggests that terpenoid compounds may play a role in mating in bumble bee queens. Terpenoid compounds were also found to play a role in territory marking and mating in bumble bee males (Bergman and Bergström 1997), although males produce predominantly low molecular weight terpenes like farnesol, whereas in queens, terpenoids are mainly represented by farnesyl esters of unsaturated fatty acids. The low volatility of these esters suggests that if they do have a signaling role, they are likely short-range signals that are perceived upon contact. Interestingly, terpenoid compounds, albeit of a different structure, were found to be the distinguishing feature of the Dufour's gland secretion of $B$. impatiens gynes (Derstine et al. 2021), where they may also serve as sex pheromones. The similarity in compounds across species, sexes and castes may point to evolutionary constrains on chemical diversity and perhaps an adoption of the same chemicals for different functions. Previous studies found that terpenoid compounds were produced by the same metabolic pathway as juvenile hormones in non-social insects (Engel et al. 2016). We know very little about the levels of juvenile hormone in bumble bee queens before and after mating and exploring the relationship between juvenile hormone level and terpenoid production (and the changes caused in these parameters by mating) would be an interesting avenue of research.

The amount and identity of wax ester components was a differentiating factor across castes. Specifically, active queens, gynes, and workers differed in the composition of non-terpenoid esters, and the differences we observed in the labial glands mirrors trends previously determined for the Dufour's gland secretions of Bombus impatiens. Workers were characterized by dodecyl esters, whereas gynes produced no dodecyl esters at all, but synthesize predominantly longer esters with 14-18 carbons in the alcohol moiety and 18-20 carbons in the acid moiety. This suggests that common biosynthetic pathways are activated in different glands, or alternatively, that esters are produced outside of the glands, possibly in the fat body, and are transported separately to different glands. Mechanisms regulating ester biosynthesis are not yet well characterized in bumble bees. The predominance of dodecyl esters in workers and octyl esters in queens of $B$. impatiens mirrors the contents from analyses of cephalic labial gland secretions of $B$. terrestris (Amsalem et al. 2014). Overall, aliphatic esters were by far, most abundant in the labial glands of active queens and QR workers, and least abundant in gynes. The common characteristic of active queens and QR workers is the fact that they were sampled from a fully functional large 
colony, unlike gynes and QL and QLBL workers, which were reared in small groups. The abundance of esters in these bees might suggest a social communication function, but, alternatively, esters might be used for their physical properties in building and repair of wax cells. Labial gland esters have been implicated in nest building in solitary bees (Kronenberg and Hefetz, 1984) but their function in social species is as yet unknown.

Hydrocarbons made up a large part of the cephalic labial gland secretions in all castes. The ratios of short- to long-chain hydrocarbons in the labial glands displayed the same trend as hydrocarbons on the cuticle, where active queens have the highest short- to long-chain hydrocarbon ratio, while gynes have the lowest. Additionally, in both gynes and workers, the change in the ratio occurs in tandem with ovarian development, and the terminal oocyte size is significantly correlated with the short- to long-chain hydrocarbon ratio. This suggests that in bumble bees, hydrocarbon synthesis is associated with oogenesis and might serve as a fertility signal, as was previously shown in solitary insects (Blomquist and Bagnères 2010).

Finally, we observed an intriguing set of unidentified relatively heavy (molecular weights 430-530 amu) compounds characterized by a base peak at $m / z=95$. The proportions of these compounds were not large (0.5$4 \%$ of total secretion) but they discriminate significantly between castes and between different treatment groups in workers, in a similar manner to esters. As with the ester components, the proportion of these compounds increased with age and their amounts significantly correlated with ester amounts. Further attempts are in progress to try and identify these compounds and understand the cause of their co-occurrence with esters.

Overall, our analysis of labial gland secretion compositions revealed differences between castes, social conditions, and physiological states in both queens and workers, and allowed us to formulate several hypotheses about the possible functions of the cephalic labial gland compounds. The terpenoid esters which are abundant in gynes may act as a sex pheromone, while the wax esters may have a social signaling function. The ratio of short- to long-chain hydrocarbons is associated or regulated by oogenesis and may signal fertility. Testing these hypotheses will require further research involving behavioral assays, and elucidation of the physiological and molecular mechanisms underlying the biosynthesis of different classes of compounds.

\section{Declarations}

Funding - This work was funded by NSF-CAREER IOS-1942127 to EA.

Conflicts of interest/Competing interests - The authors declare that they have no conflict of interest/competing interests.

Availability of data and material - Not applicable

Code availability - Not applicable

Authors' contributions - EA designed the study, MO and GV conducted the experiments, $\mathrm{MO}$ analyzed the data, $\mathrm{MO}, \mathrm{AH}$, and JGM performed the chemical identification, JGM synthesized the standards, MO and EA wrote the manuscript and all authors reviewed and approved of the final draft.

Ethics approval - Not applicable

Consent to participate - Not applicable

Page $17 / 23$ 


\section{References}

1. Afshar K, Dube FF, Najafabadi HS, Bonneil E, Thibault P, Salavati R, Bede JC (2013) Insights into the insect salivary gland proteome: diet-associated changes in caterpillar labial salivary proteins. J Insect Physiol 59:351-366. doi:10.1016/j.jinsphys.2013.01.002

2. Alford DV (1969) A study of the hibernation of bumble bees (Hymenoptera: Bombidae) in Southern England. J Anim Ecol 38:149-170

3. Amsalem E (2020) One problem, many solutions: Female reproduction is regulated by chemically diverse pheromones across insects. In: Jurenka R (ed) Advances in Insect Physiology, vol 59. Academic Press, pp 131-182. doi:https://doi.org/10.1016/bs.aiip.2020.09.001

4. Amsalem E, Grozinger CM, Padilla M, Hefetz A (2015) The physiological and genomic bases of bumble bee social behaviour. In: Amro Z, Clement FK (eds) Genomics, Physiology and Behaviour of Social Insects, vol 48. Academic Press, Adv In Insect Phys, pp 37-93

5. Amsalem E, Kiefer J, Schulz S, Hefetz A (2014) The effect of caste and reproductive state on the chemistry of the cephalic labial glands secretion of Bombus terrestris. J Chem Ecol 40:900-912. doi:10.1007/s10886014-0484-3

6. Amsalem E, Twele R, Francke W, Hefetz A (2009) Reproductive competition in the bumble-bee Bombus terrestris: do workers advertise sterility? Proceedings of the Royal Society B: Biological Sciences 276:12951304 doi:10.1098/rspb.2008.1688

7. Appelgren M, Bergström G, Svensson B, Björn C (1991) Marking pheromones of megabombus bumble bee males. Acta Chem Scand 45:972-974. doi:10.3891/acta.chem.scand.45-0972

8. Bergman P, Bergström G (1997) Scent marking, scent origin, and species specificity in male premating behavior of two scandinavian bumblebees. J Chem Ecol 23:1235-1251.

doi:10.1023/b:Joec.0000006461.69512.33

9. Bertsch A, Schweer H, Titze A, Tanaka H (2005) Male labial gland secretions and mitochondrial DNA markers support species status of Bombus cryptarum and B. magnus (Hymenoptera, Apidae). Insectes Sociaux 52:45-54 doi:10.1007/s00040-004-0761-1

10. Billen J, Šobotník J (2015) Insect exocrine glands. Arthropod Structure Development 44:399-400. doi:https://doi.org/10.1016/j.asd.2015.08.010

11. Blomquist GJ, Bagnères A-G (2010) Insect hydrocarbons: biology, biochemistry, and chemical ecology. Cambridge University Press, Cambridge

12. Derstine NT, Villar G, Orlova M, Hefetz A, Millar J, Amsalem E (2021) Dufour's gland analysis reveals caste and physiology specific signals in Bombus impatiens. Sci Rep 11:2821. doi:10.1038/s41598-021-82366-2

13. Duchateau MJ, Velthuis HHW (1988) Development and reproductive strategies in Bombus terrestris colonies. Behavior 107:186-207

14. Engel KC, Stokl J, Schweizer R, Vogel H, Ayasse M, Ruther J, Steiger S (2016) A hormone-related female antiaphrodisiac signals temporary infertility and causes sexual abstinence to synchronize parental care. Nat Commun 7:11035. doi:10.1038/ncomms11035 
15. Ghosh A, Basu A (2016) Robust estimation in generalized linear models: the density power divergence approach. Test 25:269-290. doi:10.1007/s11749-015-0445-3

16. Jarau S, Schulz CM, Hrncir M, Francke W, Zucchi R, Barth FG, Ayasse M (2006) Hexyl decanoate, the first trail pheromone compound identified in a stingless bee, Trigona recursa. J Chem Ecol 32:1555-1564. doi:10.1007/s10886-006-9069-0

17. Jarau $S$ et al (2010) Workers make the queens in melipona bees: identification of geraniol as a caste determining compound from labial glands of nurse bees. J Chem Ecol 36:565-569. doi:10.1007/s10886010-9793-3

18. Katzav-Gozansky T, Soroker V, lonescu A, Robinson GE, Hefetz A (2001) Task-related chemical analysis of labial gland volatile secretion in worker honeybees (Apis mellifera ligustica). J Chem Ecol 27:919-926. doi:10.1023/a:1010330902388

19. Keeling Cl, Plettner E, Slessor KN (2004) Hymenopteran semiochemicals. In: Schulz S (ed) The chemistry of pheromones and other semiochemicals I. Springer Berlin Heidelberg, Berlin, pp 133-177. doi:10.1007/b95452

20. Loh W-y (1987) Some modifications of levene's test of variance homogeneity. J Stat Comput Simul 28:213226. doi:10.1080/00949658708811030

21. Musser RO, Farmer E, Peiffer M, Williams SA, Felton GW (2006) Ablation of caterpillar labial salivary glands: technique for determining the role of saliva in insect-plant interactions. J Chem Ecol 32:981-992. doi:10.1007/s10886-006-9049-4

22. Orlova M, Treanore ED, Amsalem E (2020) Built to change: dominance strategy changes with life stage in a primitively eusocial bee. Behav Ecol 31(6):1361-1368

23. Poiani SB, Cruz-Landim CD (2009) Cephalic salivary gland ultrastructure of worker and queen eusocial bees (Hymenoptera, Apida). Animal Biology 59:299-311

24. Poiani SB, Cruz-Landim Cd (2010) Cephalic salivary glands of two species of advanced eusocial bees (Hymenoptera: Apidae): morphology and secretion. Zoologia (Curitiba) 27:979-985. doi:10.1590/s198446702010000600021

25. Reinhard J, Kaib M (1995) Interaction of pheromones during food exploitation by the termite Schedorhinotermes lamanianus. Physiol Entomol 20:266-272. doi:https://doi.org/10.1111/j.13653032.1995.tb00011.x

26. Rivera-Vega LJ, Stanley BA, Stanley A, Felton GW (2018) Proteomic analysis of labial saliva of the generalist cabbage looper (Trichoplusia ni) and its role in interactions with host plants. J Insect Physiol 107:97-103. doi:10.1016/j.jinsphys.2018.03.001

27. Sehnal F, Sutherland T (2008) Silks produced by insect labial glands. Prion 2:145-153. doi:10.4161/pri.2.4.7489

28. Sillam-Dussès D et al (2012) Comparative study of the labial gland secretion in termites (Isoptera). Plos One 7:e46431. doi:10.1371/journal.pone.0046431

29. Stangler ES, Jarau S, Hrncir M, Zucchi R, Ayasse M (2009) Identification of trail pheromone compounds from the labial glands of the stingless bee Geotrigona mombuca. Chemoecology 19:13-19. doi:10.1007/s00049-009-0003-0 
30. Starkey J, Brown A, Amsalem E (2019) The road to sociality: brood regulation of worker reproduction in the simple eusocial bee Bombus impatiens. Anim Behav 154:57-65. doi:10.1016/j.anbehav.2019.06.004

31. Stokl J, Steiger S (2017) Evolutionary origin of insect pheromones. Current Opinions Insect Sciences 24:3642. doi:10.1016/j.cois.2017.09.004

32. Svensson BG, Bergström G (1977) Volatile marking secretions from the labial gland of North European pyrobombus D. T. males (Hymenoptera, Apidae). Insectes Soc 24:213-224. doi:10.1007/BF02227172

33. Valterova I, Martinet B, Michez D, Rasmont P, Brasero N (2019) Sexual attraction: a review of bumblebee male pheromones. Zeitschrift für Naturforschung C 74:233-250. doi:10.1515/znc-2019-0003

34. Yau K, Kuk A (2002) Robust estimation in generalized linear mixed models. Journal of the Royal Statistical Society Series B 64:101-117. doi:10.1111/1467-9868.00327

\section{Figures}
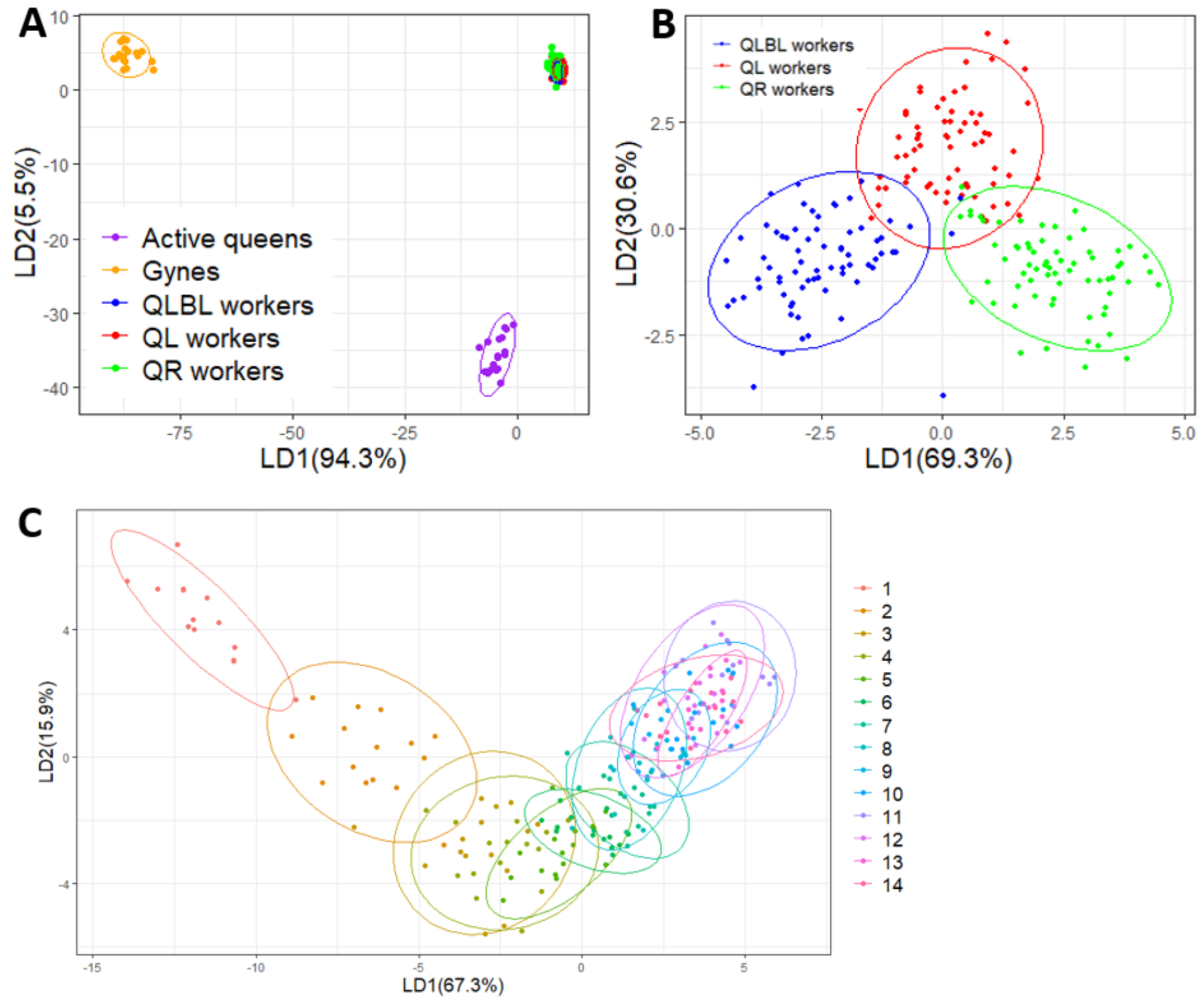


\section{Figure 1}

Discriminant analysis based on the relative proportions of the cephalic labial gland components considering caste (A), worker treatment (B), and worker age (C) as grouping variables. The plots display the first two discriminant functions, with percentage of explained variance in parentheses.
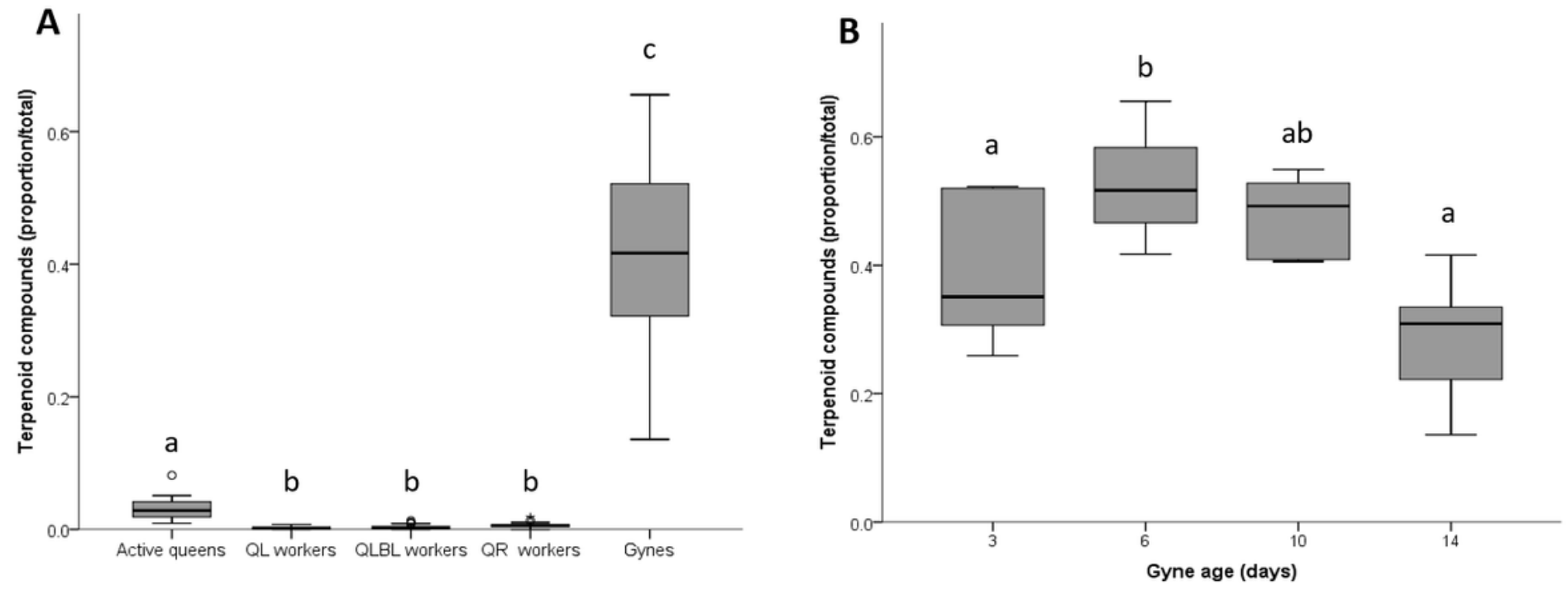

\section{Figure 2}

Relative proportions of terpenoid compounds in bees of different caste and treatment group (A), and in gynes of different age (B). Letter above columns denote significant differences at $a=0.05$. 


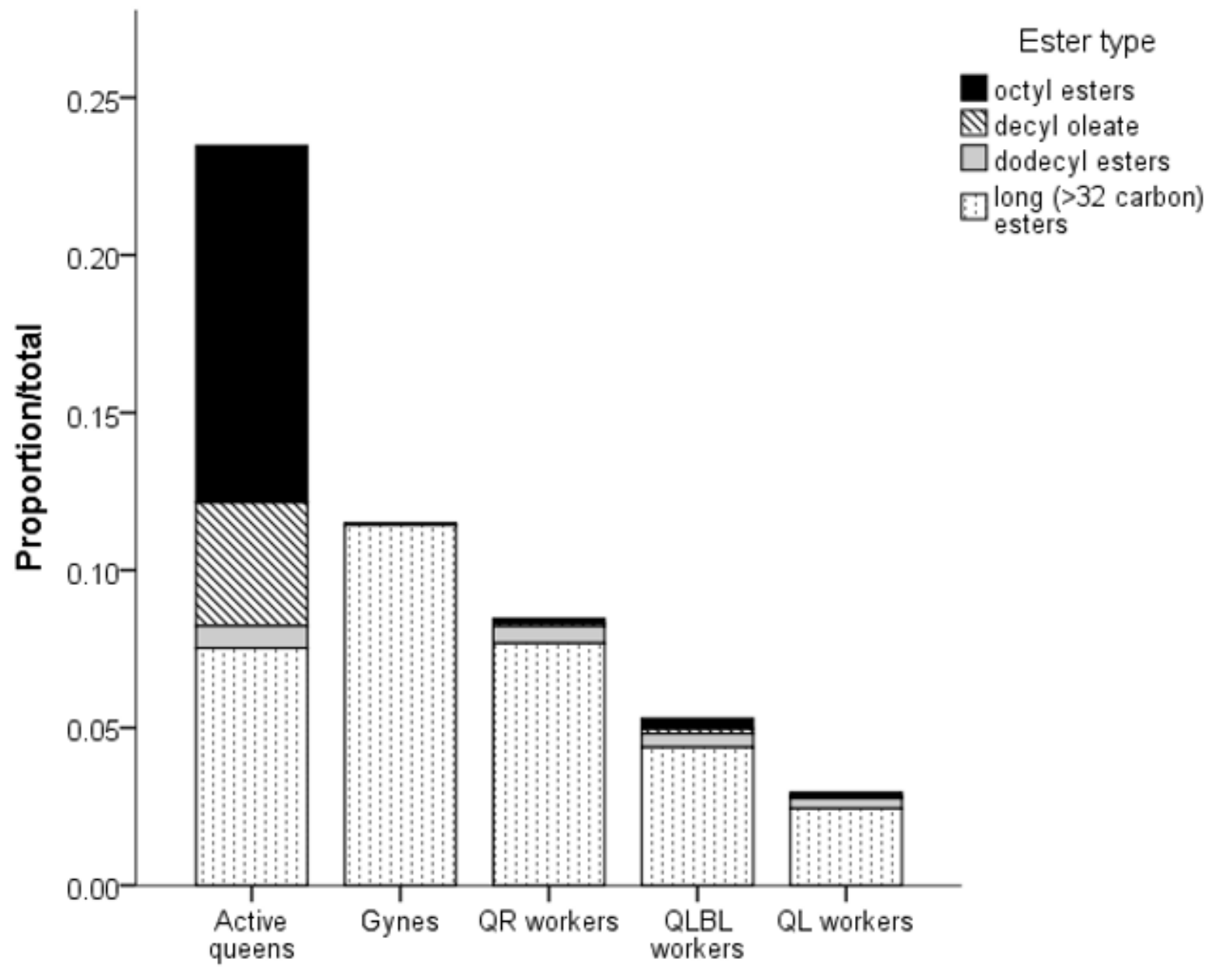

\section{Figure 3}

Relative proportions of wax (aliphatic) ester components in bees of differing caste and treatment groups. Bar height represents proportion of total secretion, and color section height represents proportions of different classes of esters. 


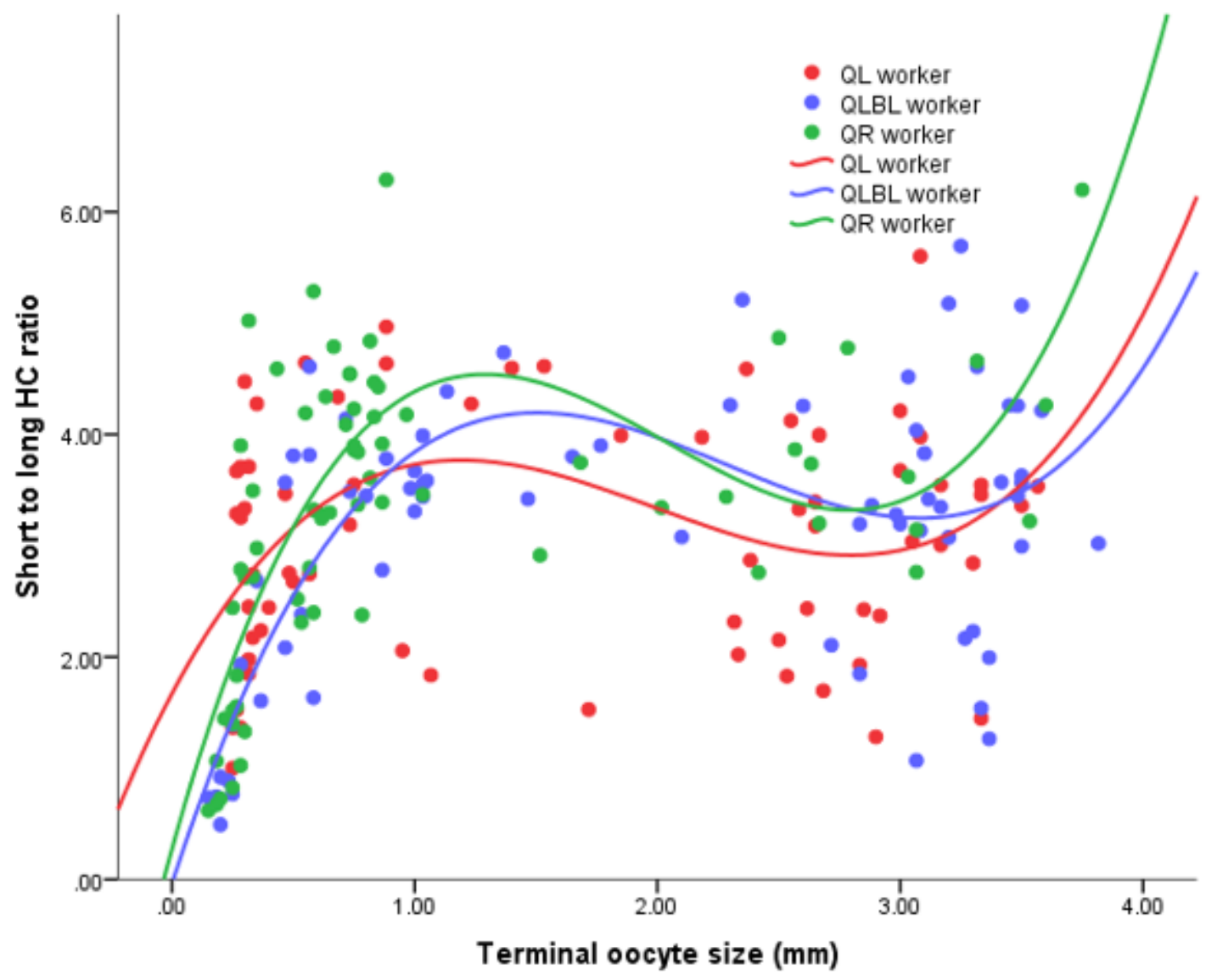

Figure 4

Relationship between the terminal oocyte size and the short- to long-chain hydrocarbon ratio in workers of different treatment groups. All trendlines were fitted by polynomial regression.

\section{Supplementary Files}

This is a list of supplementary files associated with this preprint. Click to download.

- TableS1.xlsx 